\title{
Access of cosmic rays to an ICME from external field lines
}

\author{
T. Laitinen ${ }^{a, *}$ and S. Dalla ${ }^{a}$ \\ a Jeremiah Horrocks Institute, \\ University of Central Lancashire, Preston, UK \\ E-mail: tlmlaitinen@uclan.ac.uk
}

Interplanetary coronal mass ejections (ICMEs) cause decreases, so-called Forbush decreases, in the cosmic ray (CR) intensities. FDs are seen as up to $25 \%$ decreases in neutron monitor counts at Earth, lasting up to over a week. An ICME is thought to cause a FD through two mechanisms: by enhancing diffusion in the ICME shock wave sheath; and by preventing the CRs from penetrating the magnetic flux rope embedded in the ICME. CR propagation during a FD is usually modelled as enhanced diffusion either within the whole ICME or within the embedded flux rope. However, a question that is so far unanswered is how the CRs can reach the isolated flux rope field lines from the open, external interplanetary field lines. We study the propagation of CRs from external field into a flux rope by employing full-orbit particle simulations with scattering. The interface between the internal and external field lines is modelled analytically. We find that the CRs can access the flux rope rapidly through $x$-point region, where the external magnetic field partially cancels the magnetic field of the flux rope. The access is rapid compared to diffusive radial propagation of CRs within the rope. We find that CR propagation within the flux rope can be modelled using diffusion models, without need to separately model the access to the isolated field lines, provided that the bounds of the diffusion area are taken as that of the isolated field lines instead of the region with smoothly rotating magnetic field. Thus, to evaluate the role of a flux rope in FDs, the extent of the region where the rope magnetic fields are not connected to the external field must be analysed.

$37^{\text {th }}$ International Cosmic Ray Conference (ICRC 2021)

July 12 th - 23rd, 2021

Online - Berlin, Germany

\footnotetext{
*Presenter
} 


\section{Introduction}

Cosmic ray $(\mathrm{CR})$ propagation in the heliosphere is guided by the interplanetary magnetic field, which is typically of Parker spiral shape. During solar eruptions, magnetic clouds that form the core of the interplanetary coronal mass ejections (ICMEs) propagate through the interplanetary medium, and their magnetic structure and enhanced turbulence affect the propagation of both the Solar Energetic Particles (SEPs) and the cosmic rays that have their origin outside of the heliosphere.

In this study, we investigate how the magnetic clouds affect the propagation of CRs. The effect is often seen in Earth-based neutron monitors as a intensity decrease of up to 25\%, the so-called Forbush Decrease (FD) [1-4]. The decrease can take up to over a week, typically with decay lasting for hours, after which recovery for up to over a week. The slow component often is accompanied with a faster, one-day symmetric decay-recovery component[5]. The faster component is attributed to the magnetic flux rope-like structure that presents a barrier for the CRs that would have to propagate across the mean field direction to reach the axis of the flux rope $[3,5]$.

The effect of the flux rope on the CR intensities is typically modelled as diffusion, with the diffusion coefficient fitted from observations to be of order $\kappa_{\text {radial }} \sim 10^{19}-10^{21} \mathrm{~cm}^{2} / \mathrm{s}($ e.g. [6, 7]). These studies model the propagation of particles as diffusion from the surface of the fluxrope towards its axis to fill the rope. However so far the magnetic connection from the external magnetic field to the isolated flux rope magnetic field lines has not been investigated. Thus, it has not been clear whether there is a need to have an additional step to model before the propagation of CRs inside the flux rope begins.

In this study, we investigate this issue by simulating CR propagation using full-orbit particle simulations. We model the flux rope, and its connection to the external magnetic field, using an analytical model, and evaluate the propagation of CRs from the external field lines to the isolated magnetic fields of the flux rope. In order to ascertain the the validity of the diffusion-based models, we compare the full-orbit simulation results with a solution of radial diffusion equation.

It should be noted that we only investigate the effect of access of particles to the isolated magnetic field lines. To that end, our flux rope model is a simple Gold-Hoyle[8] cylindrical flux rope embedded in a constant magnetic field, with no changes in the size or internal magnetic field as a function of time included. With this approach, we can isolate the effects related to the magnetic connection between the external and the isolated internal magnetic fields on the CR propagation into the flux rope.

We present the models in Section2, give our results in Section 3, and discuss their significance, drawing conclusions in Section 4. Further details of the study, and further discussion, can be found in [9].

\section{Models}

The flux rope in our study is modelled using the force-free Gold-Hoyle model[8] which in $(r, \phi, Z)$, is

$$
\mathbf{B}_{\mathrm{GH}}=\frac{B_{a} b r}{b^{2} r^{2}+1} \hat{\mathbf{e}}_{\phi}+\frac{B_{a}}{b^{2} r^{2}+1} \hat{\mathbf{e}}_{\mathbf{Z}}
$$




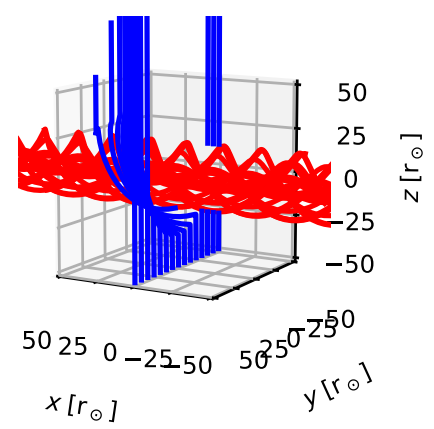

Figure 1: 3D projection of the flux rope magnetic field, with $r_{b}=32 \mathrm{r}_{\odot}, t_{b}=1 \mathrm{r}_{\odot}$ and $B_{a}=5 B_{0}$. The red curves show the field lines that are isolated from the open field (blue curves).

where $B_{a}$ gives the axial field, and $b$ parametrises the azimuthal winding of the field around the axis. We limit he flux rope with a modulating function

$$
f(s)=2 s^{3}-3 s^{2}+1
$$

which goes from 1 to 0 as the variable $s$ goes from 0 to 1 , and has a vanishing derivative at 0 and 1 , and define the flux rope field as

$$
\mathbf{B}_{r}= \begin{cases}\mathbf{B}_{\mathrm{GH}}(r) & r<r_{b} \\ f\left(\frac{r-r_{b}}{t_{b}}\right) \mathbf{B}_{\mathrm{GH}} & r_{b} \leq r<r_{b}+t_{b} \\ 0 & r \geq r_{b}+t_{b},\end{cases}
$$

where $r_{b}$ is the rope radius $r_{b}$ and $t_{b}$ the thickness of a sheath region around the flux rope. This modulation ensures that the extent of the flux rope is finite, as implied by observations[10]

The magnetic flux rope is embedded in an external magnetic field, which we take as constant $B_{0}$. Thus, the total magnetic field in our simulations will be

$$
\mathbf{B}=\mathbf{B}_{\mathrm{r}}+\mathbf{B}_{0}
$$

We simulate the CR propagation using full-orbit test particle simulation code[11]. The CRs are scattered with velocity isotropisation events as a Poisson process characterised by scattering time $\tau_{\mathrm{sc}}=\lambda_{\mathrm{sc}} / v$. The parallel scattering mean free path $\lambda_{\mathrm{sc}}$ is kept constant in the simulations, likewise the velocity $v$ is constant.

In addition to the full-orbit simulations, we model solve the CR propagation into a flux rope using a 1D radial diffusion equation for density $n(r, t)$

$$
\frac{\partial n(r, t)}{\partial t}=\frac{1}{r} \frac{\partial}{\partial r} r \kappa_{\text {radial }}(r) \frac{\partial n}{\partial r} .
$$

where the diffusion coefficient is given as $[9,12]$

$$
\kappa_{\text {radial }}=\frac{r_{L}^{2}}{3 \tau_{\mathrm{sc}}} .
$$




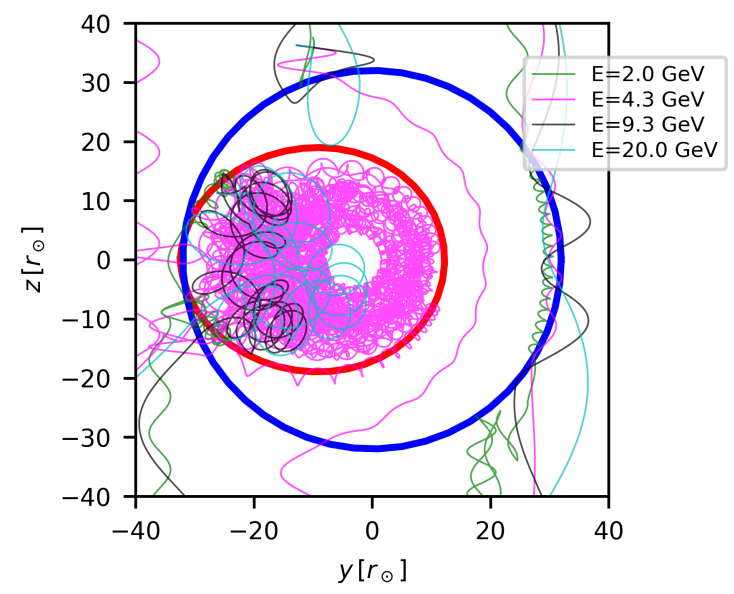

Figure 2: A sample of particle paths in the field configuration shown in Figure 1. The flux rope radius $r_{b}=32 r_{\odot}$ is shown by the blue circle, and the isolated field region with the red dashed oval. The parallel scattering mean free path is $0.3 \mathrm{AU}$.

The diffusion equation is solved using the Crank-Nicolson scheme, with initial conditions given as constant density at the outer boundary of the cylinder, and the cylinder initially empty. Further details on the scheme can be found in [9].

\section{Results}

We used the full orbit particle simulations to investigate particle access to the flux rope structure for various energies and scattering parameters. The flux rope parameters are fixed to $r_{b}=32 \mathrm{r}_{\odot}$, $t_{b}=1 \mathrm{r}_{\odot}, B_{a}=5 B_{0}$, and $B_{0}=5 \mathrm{nT}$. To demonstrate the different types of particle orbits, we show sample tracks of particles in Figure 2. The thick blue solid curve shows the outer boundary of the flux rope, and the solid red curve the region of the flux rope that is not connected to the external field lines. The particle orbits can be divided to three categories: those that do not enter the blue circle but are reflected from the stronger magnetic field, those that pass through the blue circle and exit from the other side, and those that enter the isolated flux rope field lines, to be trapped, and propagating further within the structure. The third category is responsible of filling the flux rope. As can be seen, the particles entering the flux rope do so preferentially close to the magnetic $\mathrm{x}$-point, or $\mathrm{x}$-line, at around $x=-32 \mathrm{r}_{\odot}, y=0$, with higher energy particles having wider range of locations to access the flux rope.

Figure 3 demonstrates how the particles gradually have access towards the flux rope axis, for $200 \mathrm{MeV}$ protons within 3.44 days. Initially the flux rope is empty, but as it starts to fill, with the combined effect of penetrating through the weak magnetic field area near the x-point, the particles quickly isotropise along the magnetic field lines within the flux rope. As a result, the density equicontours are ordered as the magnetic field equipotential surfaces, which are asymmetric 


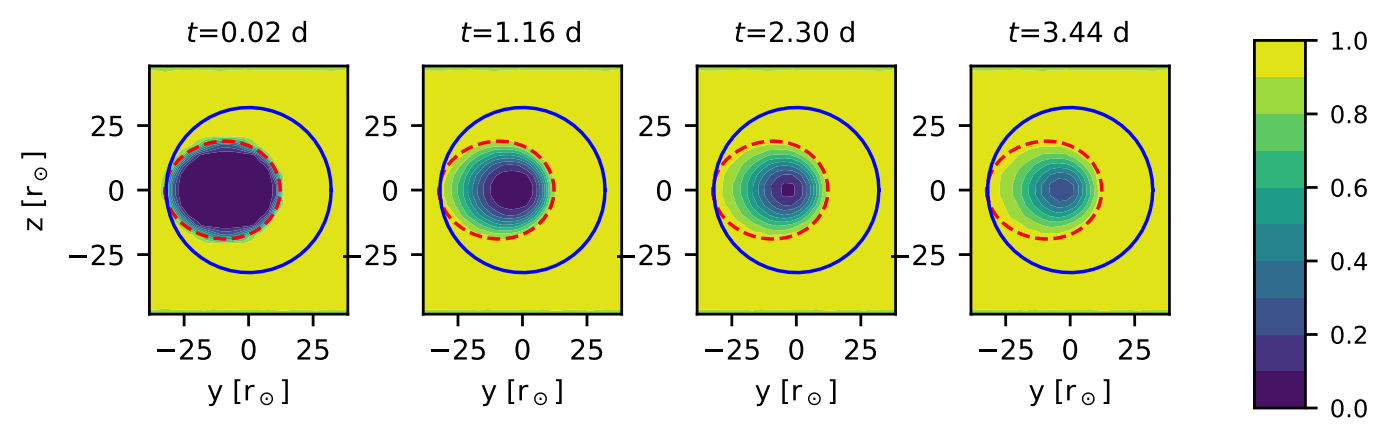

Figure 3: Density contours of $200 \mathrm{MeV}$ protons inside and around the modelled flux rope $\left(\lambda_{\mathrm{sc}}=0.1 \mathrm{au}\right)$. The red dashed curve shows the region of isolated field lines, and the solid blue line curve the extent of the rope.

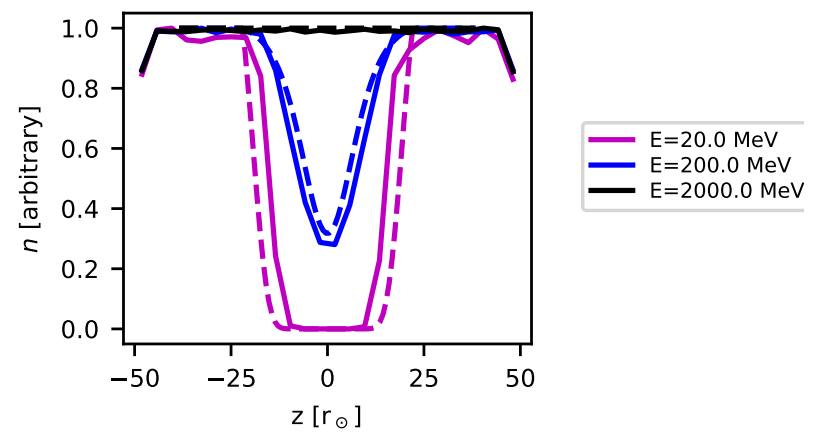

Figure 4: Density of $200 \mathrm{MeV}$ protons across a flux rope obtained from the full-orbit simulations (solid curves) and the radial diffusion solution (dashed curves) 3 days after injection.

with respect to the flux rope axis. Further analysis with different particle energies and transport parameters are shown in [9].

We next investigated whether the access of CRs to the isolated magnetic field lines of the flux rope constistute a significant hindrance that would need to be taken into account when modelling FDs. To do that, we compared the full-orbit simulation results with a simple diffusion model with radial diffusion of particle density into the flux rope, as given by Equation (5). The diffusion coefficient was selected to be consistent with the full-orbit simulations with the use of Equation (6), and the flux rope radius was set to be the extent of the isolated flux rope region as depicted in Figure 3 by the red dashed curve, at $x=0$.

We show the result of the comparison in Figure 4, for different scattering mean free paths, for protons of $200 \mathrm{MeV}, 3$ days after injection. The full-orbit density profiles are obtained at the $x=0$-plane, and are shown with the solid curves. The dashed curves show the corresponding result for the radial diffusion solution. As can be seen, the two approaches give almost exactly the same result, with the small differences likely to be caused by the full-orbit model being 2.5 -dimensional 
and the diffusion model 1-dimensional. Further comparisons are shown in [9].

\section{Discussion and Conclusions}

In this study, we investigated in detail how cosmic rays can access the isolated magnetic field lines of a flux rope from the external field lines. We used a simple analytical flux rope model embedded in an external magnetic field, which resulted in a configuration where the magnetic connection to the isolated fields is facilitated only through an x-point, or $\mathrm{x}$-line. For approaches with infinitesimal particle Larmor radius, or guiding centre approximations, this access point would be singular. However, with finite Larmor radius, higher-energy particles have larger practical area where they can penetrate into the isolated field lines (Figure 2. Once inside the flux rope, the particles can diffuse inwards to the inner magnetic field lines, as they scatter in velocity space, which results in random walk of their gyrocentres.

The question is then whether the access through the vicinity of the x-point is fast enough so that we could justify using the diffusion approach for modelling Forbush decreases within the flux rope. Our simulation results suggest that this is indeed the case.

Our result is dependent on the turbulence conditions, particularly in the region near the x-point that separates the external and internal magnetic field. We have not modelled the strongly turbulent sheath region (e.g. [13]). However, the diffusion coefficients are proportional to the turbulence strength relative to the mean magnetic field, which does not change significantly from the solar wind to the sheath[13], implying that the significance of the sheath region may be small. Within the flux rope the relative turbulence strength is weaker, but still can contribute to random-walk of field lines. However, as shown by [14], the random-walk of field lines tends to follow the 2D equipotential contours and hence are not likely to enhance the propagation of the CRs towards across the flux rope fields, towards the flux rope axis.

Our simple model was kept simple in purpose, to exclude effects due to, e.g. external curvature of the flux rope (e.g. [15]) that result in drifts that affect particle motion, and changes in the flux rope size and magnetic field (e.g. [7]), which will also affect the particle energies[16]. Incorporating these effects into the full-orbit modelling in the future will inform us about the finite Larmor radius consequences that may need to be taken into account when using simple models.

\section{References}

[1] S.E. Forbush, On the Effects in Cosmic-Ray Intensity Observed During the Recent Magnetic Storm, Physical Review 51 (1937) 1108.

[2] J.A. Lockwood, Forbush Decreases in the Cosmic Radiation, Space Sci. Rev. 12 (1971) 658.

[3] H.V. Cane, Coronal Mass Ejections and Forbush Decreases, Space Sci. Rev. 93 (2000) 55.

[4] I.G. Richardson and H.V. Cane, Galactic Cosmic Ray Intensity Response to Interplanetary Coronal Mass Ejections/Magnetic Clouds in 1995 - 2009, Sol. Phys. 270 (2011) 609. 
[5] L.R. Barnden, The Large-Scale Magnetic Field Configuration Associated With Forbush Decreases, in International Cosmic Ray Conference, vol. 2 of International Cosmic Ray Conference, p. 1277, Jan, 1973.

[6] H.V. Cane, I.G. Richardson and G. Wibberenz, The Response of Energetic Particles to the Presence of Ejecta Material, in International Cosmic Ray Conference, vol. 4 of International Cosmic Ray Conference, p. 377, Jan, 1995.

[7] M. Dumbović, B. Heber, B. Vršnak, M. Temmer and A. Kirin, An Analytical Diffusion-Expansion Model for Forbush Decreases Caused by Flux Ropes, ApJ 860 (2018) 71 [1805.00916].

[8] T. Gold and F. Hoyle, On the origin of solar flares, MNRAS 120 (1960) 89.

[9] T. Laitinen and S. Dalla, Access of Energetic Particles to a Magnetic Flux Rope from External Magnetic Field Lines, ApJ 906 (2021) 9.

[10] L. Burlaga, E. Sittler, F. Mariani and R. Schwenn, Magnetic loop behind an interplanetary shock: Voyager, Helios, and IMP 8 observations, J. Geophys. Res. 86 (1981) 6673.

[11] S. Dalla and P.K. Browning, Particle acceleration at a three-dimensional reconnection site in the solar corona, A\&A 436 (2005) 1103.

[12] E.N. Parker, The passage of energetic charged particles through interplanetary space, Planet. Space Sci. 13 (1965) 9.

[13] J.J. Masías-Meza, S. Dasso, P. Démoulin, L. Rodriguez and M. Janvier, Superposed epoch study of ICME sub-structures near Earth and their effects on Galactic cosmic rays, A\& A 592 (2016) A118 [1605 .08130].

[14] M.C. Ghilea, D. Ruffolo, P. Chuychai, W. Sonsrettee, A. Seripienlert and W.H. Matthaeus, Magnetic Field Line Random Walk for Disturbed Flux Surfaces: Trapping Effects and Multiple Routes to Bohm Diffusion, ApJ 741 (2011) 16.

[15] W. Krittinatham and D. Ruffolo, Drift Orbits of Energetic Particles in an Interplanetary Magnetic Flux Rope, ApJ 704 (2009) 831.

[16] K. Munakata, S. Yasue, C. Kato, J. Kota, M. Tokumaru, M. Kojima et al., On the Cross-Field Diffusion of Galactic Cosmic Rays into an ICME, in Advances in Geosciences, Volume 2: Solar Terrestrial (ST), vol. 2, p. 115, Jan, 2006, DOI. 